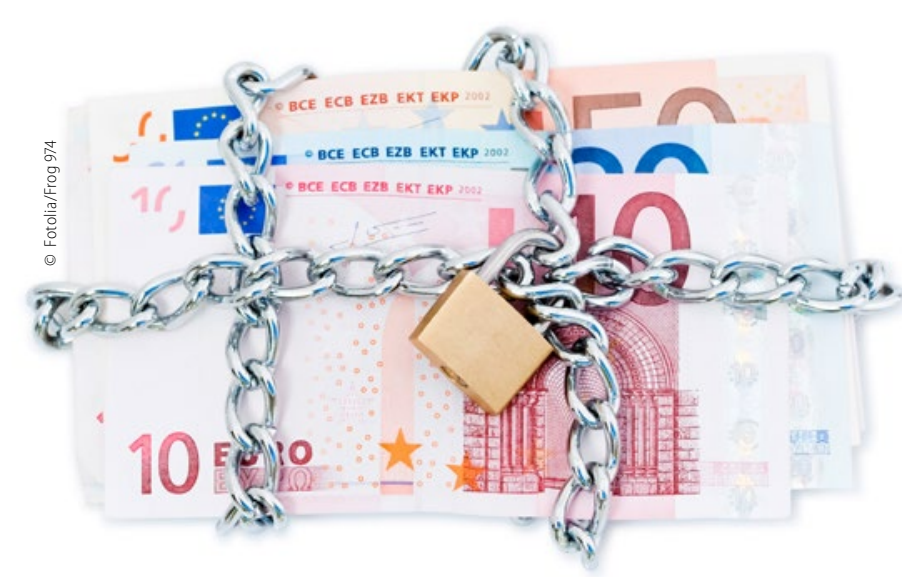

\title{
Selbstbestimmung - ein elementares Recht in der gesundheitlichen Versorgung
}

Liebe Kolleginnen und Kollegen,

bei der Konstituierenden Vertreterversammlung (VV) mit Wahl des neuen Vorstandes der Kassenzahnärztlichen Bundesvereinigung (KZBV) in Berlin wurde deutlich, welche nachhaltigen Auswirkungen das GKV-Selbstverwaltungsstärkungsgesetz hat. Das Diktat, vielmehr das Oktroyieren der Inhalte der Dienstverträge der neuen Vorstandsmitglieder durch die Aufsichtsbehörde kommt einer Beseitigung der Entscheidungskompetenzen der VV gleich, in der die Kassenzahnärztlichen Vereinigungen der Länder vertreten sind.

Die vorgelegte Vertragsfassung macht es insbesondere freiberuflichen Zahnärzten, die noch in der Praxis tätig sind, schwer bis fast unmöglich, im Vorstand der KZBV zu arbeiten. Es wird einer Entwicklung Vorschub geleistet, die es erschwert, dass freiberuflich tätige Zahnärzte ihren zahnärztlichen Sachverstand in die Selbstverwaltungsgremien einbringen. Gleichzeitig bedeutet die Vertragsfassung eine Besserstellung und damit Einflussnahme von fachlich nicht qualifizierten Bürokraten auf die Entscheidungsgremien der KZBV als Selbstverwaltungskörperschaft.

\section{Wichtiger Bestandteil einer lebendigen Demokratie}

Bei aller Befähigung dieser Beteiligten: Als Selbstverwaltung definiert die Rechtswissenschaft die „Übertragung von Verwaltungsaufgaben an rechtlich verselbstständigte Organisationen. Damit werden die Bürger unmittelbar an der Erfüllung staatlicher Aufgaben beteiligt. Selbstverwaltung ist damit ein grundsätzlich wichtiger Bestandteil einer lebendigen Demokratie und ermöglicht den Betroffenen eine eigenverantwortliche Mitgestaltung (Subsidiaritätsprinzip)“. Die Betroffenen sind wir Zahnärzte, warum wird unsere Beteiligung an den Gremien der KZBV von der Aufsicht erschwert?

Das Selbstverwaltungsstärkungsgesetz ist ein Selbstverwaltungsvernichtungsgesetz, ganz zu Gunsten einer politisch gewollten Staatsmedizin. Ist Selbstverwaltung im Sinne von Selbstbestimmung für die Zahnärzteschaft damit noch möglich?

Wir, die Zahnärzte, haben alleine die fachliche Kompetenz und stellen die zahnärztliche Versorgung in Deutschland. Wir haben eine Chance zur Selbstbestimmung, wenn wir - egal ob Zahnärztin oder Zahnarzt, jung oder alt, selbstständig oder angestellt, Stadt oder Land - als Wir zusammenstehen. Die zahnärztliche Versorgung in Deutschland nimmt - dank unserer täglichen Arbeit - weltweit einen Spitzenplatz ein. Grund genug, um mit Selbstbewusstsein das durch das freiberufliche Prinzip und nicht durch staatliche Bürokratie Erreichte fortzuführen.

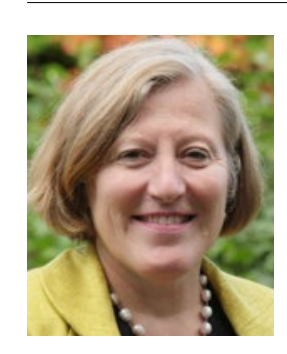

Dr. Gudrun Kaps-Richter

Stellvertretende FVDZ-Bundesvorsitzende

\section{Spielregeln berufsintern festlegen}

Wir stehen vor der Entscheidung, ob wir unseren zahnärztlichen Beruf in Zukunft als "Maker" oder "Taker" ausüben werden. Wir müssen die Spielregeln von der Ausbildung über die Leistungsinhalte bis zur Honorierung unserer Leistungen berufsintern festlegen können, und diese dann in die Politik hineintragen können, damit eine vernünftige fachlich kompetente Umsetzung ermöglicht wird. Es geht hier nicht ausschließlich um die Selbstbestimmung eines Berufsstandes, letztendlich geht es auch um die Selbstbestimmung unserer Patienten. Deren Souveränität - sowohl bei der Therapiewahl als auch bei der Eigenverantwortung für ihre Gesundheit - muss durch unser Handeln gestärkt werden. Im Mittelpunkt steht für jeden Arzt und Zahnarzt der Patient. Die Zahngesundheit unserer Patienten ist unsere zentrale Aufgabe. Der Patient wird zu oft über all der Bürokratie vergessen. Wir erleben zurzeit wiederholt bei Wahlen, was es bedeutet, wenn Menschen sich vergessen fühlen. Die Frage ist nicht, ob Selbstbestimmung möglich ist, ganz einfach, weil sie nötig ist und ein elementares Recht in der gesundheitlichen Versorgung sein muss, um des Patienten willen. Wir müssen die Weiterentwicklung aufmerksam beobachten, insbesondere die Spielräume juristischer Interpretationen des GKV-Selbstversorgungsstärkungsgesetzes. Aber wir werden prüfen müssen, wie wir unsere Selbstbestimmung selbst mitgestalten - auch und vor allem zum Wohle unserer Patienten. Es muss nicht Schluss sein mit der Freiberuflichkeit - im Sinne des Grundgesetzes und mündiger Bürger einer lebendigen Demokratie: Selbstbestimmung, Freiberuflichkeit first!

Ihre<smiles>CCCCC(=O)COC</smiles>

Dr. Gudrun Kaps-Richter 